\title{
A Four-Drug Induction Therapy Including Raltegravir for the Treatment of Naïve HIV-Infected Patients
}

\author{
Katia Falasca ${ }^{1 *}$, Claudio Ucciferri ${ }^{1,2}$, Marta Di Nicola ${ }^{3}$, Francesca Vignale ${ }^{1}$, Delia Racciatti ${ }^{1}$ and Jacopo Vecchiet \\ ${ }^{1}$ Clinic of Infectious Diseases, Department of Medicine and Science of Aging, University “G. d'Annunzio" Chieti-Pescara, Italy \\ ${ }^{2}$ Department of Medicine and Health Sciences, University of Molise, Campobasso, Italy \\ ${ }^{3}$ Laboratory of Biostatistics, Department of Experimental and Clinical Sciences, University "G. d'Annunzio" Chieti-Pescara, Italy
}

\begin{abstract}
Background: Despite the recent advances in antiretroviral therapy, human immunodeficiency virus type 1 (HIV-1) remains a global health threat. The aim of this study was evaluated the efficacy of a four-drug induction antiretroviral therapy (ART), including raltegravir (RAL), on the rapidity of suppression of plasma HIV-RNA below detectable concentrations and its impact on immunological recovery.
\end{abstract}

Methods: In this single centre, randomized prospective trial, 32 naïve HIV+ patients at the same clinical stage were enrolled and randomized for baseline viral load: sixteen subjects started ART with four antiretroviral drugs including RAL, while the remaining patients started the same therapy without RAL. Viro-immunological and metabolic parameters, indexes of hepatic and renal functionality were measured at baseline (T0) and after three (T3), six (T6) and twelve months (T12) from therapy introduction.

Results: We observed a faster viral drop in the group under RAL-therapy with respect to the other group. At the first month (T1) of therapy, the HIV-RNA was significantly lower in the patients receiving RAL-therapy $(p<0.05)$. Immunological recovery was higher in patients with RAL than in those on other therapy at all detection times, with a significant increase at $\mathrm{T} 3, \mathrm{~T} 6$ and $\mathrm{T} 12(\mathrm{p}=0.02)$.

Conclusion: In this study, we found, for the first time, a rapid and significant improvement in CD4+ T cells count in patients with four drug induction therapies. The four-drug regimen was safe, well tolerated and also associated with a rapid decay of plasma HIV-RNA levels.

Keywords: Raltegravir; Induction; HIV; Intensification; Immunological recovery

\section{Background}

The primary goals of anti-HIV therapy are the suppression of plasma viral replication and the persistent recovery of number of circulating CD4+ T cells, the main target of HIV [1,2]. Highly active antiretroviral therapy achieves these goals for many patients, resulting in a reduction of HIV-associated morbidity and mortality with an estimated survival similar to the normal population [3]. The choice of the initial therapy is crucial to ensure a good long-term management of HIV infection [4], therefore optimization of the initial therapy represents a cornerstone to gain clinical and economic outcomes, including improvement of immune function and quality of life, and ability to control other comorbidities. There are observations that support the utility of intensified antiretroviral therapeutic (ART) regimens in accelerating the suppression of viral load and limiting the development of drug resistances [5]. In the past years, first-line treatments with 4,5 or more antiretrovirals did not produce better virological outcomes than the standard 3 drugs-based ART. Indeed, evolutionary studies in macaque models [6] have suggested that viral replication may be completely blocked by therapy and that development of ART drug resistance was unlikely to occur during the initial viremia decay, suggesting that treatments were quite active, and resistant variants were not pre-existing.

On the other hand, previous studies have shown a correlation between a higher resistance selection and a longer time to reach an undetectable viremia $[7,8]$. In recent years, the integrase inhibitors, a new class of antiretrovirals, has been introduced in clinical practice preventing a critical step in HIV 1 replication [9]. Of these, raltegravir (RAL) represents the first integrase inhibitor available in the armamentarium against HIV [10], providing a rapid viremia suppression when combined with an optimized backbone treatment [11]. Furthermore, a recent study [12] showed a fully active salvage antiretroviral therapy including RAL is able to completely block the HIV-1 replication in plasma.

The first observation of the efficacy and tolerability of RAL was reported in the STARTMRK trial in naive HIV positive patients, where RAL was compared with efavirenz (EFV) in combination with tenofovir (TDF) and emtricitabine (FTC). The RAL group showed a viral regimen suppression $(<50$ copies $/ \mathrm{ml})$ at week 48 in the $86.1 \%$ of cases versus $81.9 \%$ of cases of the EFV group. Therefore, an initial ART including an integrase inhibitor or RT inhibitor could ensure a better decay of virus production [13]. As a result, $\mathrm{RAL}+(\mathrm{TDF}+\mathrm{FTC})$ actually represents a possible useful alternative first-line [14]. Furthermore, recent evidences suggested that an early suppression of plasma HIVRNA below detectable concentrations could be a positive predictor on the long term virological suppression and could prevent virological failure [15]. The immunological recovery plays also a crucial role in preventing the clinical progression of the disease [16].

${ }^{*}$ Corresponding author: Prof. Katia Falasca, Clinic of Infectious Diseases, Dept of Medicine and Science of Aging, "G. d'Annunzio" University, School of Medicine, Via dei Vestini, 66013 Chieti, Italy; Tel: +39 0871 357582; Fax +39 0871 358595; E-mail:k.falasca@unich.it

Received June 20, 2017; Accepted June 24, 2017; Published June 29, 2017

Citation: Falasca K, Ucciferri C, Nicola MD, Vignale F, Racciatti D, et al. (2017) A Four-Drug Induction Therapy Including Raltegravir for the Treatment of Naïve HIV-Infected Patients. J AIDS Clin Res 8: 705. doi: 10.4172/2155-6113.1000705

Copyright: @ 2017 Falasca K, et al. This is an open-access article distributed under the terms of the Creative Commons Attribution License, which permits unrestricted use, distribution, and reproduction in any medium, provided the original author and source are credited. 


\section{Methods}

The aim of this study was to evaluate the effectiveness of a four-drug induction cART including RAL, analyzing the rapidity of the viral load reduction from baseline and its impact on immune recovery.

This was a single-centre, randomized, prospective trial realized at the Clinic of Infectious Diseases of "SS. Annunziata" Hospital, "G. d'Annunzio" University of Chieti-Pescara (Italy). Patients were enrolled from January 2011 to December 2013.

The study population included $32 \mathrm{HIV}+$ subjects naïve for any treatment; of these, sixteen patients started ART with four antiretroviral drugs including RAL (group 1), while the other patients started the same triple therapy without RAL (group 2).

Inclusion criteria were: Caucasian ethnicity, HIV infection with HIV-RNA $>1000$ copies/ml, naïve to cART and neither acute nor recent HIV infection according to medical history and positivity of antibodies against HIV. Exclusion criteria were: current alcohol or drug abuse; use of megestrol acetate, ketoconazole, steroids, growth hormone, medroxyprogesterone acetate, testosterone, or any anabolic agent in the 6 months prior to study enrolment; any acute infection in the 6 months prior to the study; acute hepatitis at the beginning of the study or advanced liver disease; kidney disease or serum creatinine level higher than twice the normal upper limit. Patients were also excluded from the study if they showed resistance to two or more drug classes and/ or resistance to the class of integrase inhibitors. In addition, patients divided in according to baseline HIV viral load $>$ or $<500000$ copies/ $\mathrm{ml}$ and the clinical stage according to CDC classification.

\begin{tabular}{|l|c|c|c|c|}
\hline \multirow{2}{*}{ Variable } & Overall & \multicolumn{2}{|c|}{ Treatment group } & \multirow{2}{*}{ p-value } \\
\cline { 2 - 4 } & $\mathbf{( n = 3 2 )}$ & HAART+RAL & HAART & \\
\cline { 2 - 4 } & & $\mathbf{( n = 1 6 )}$ & $\mathbf{( n = 1 6 )}$ & \\
\hline Gender, Male/Female & $24 / 8$ & $12 / 4$ & $12 / 4$ & $0.657^{b}$ \\
\hline Age (yr), mean $\mathbf{n} \mathbf{S}$ & $43.6 \pm 11.7$ & $43.4 \pm 11.9$ & $43.9 \pm 11.9$ & 0.744 \\
\hline CDC stage, $\boldsymbol{n}(\%)$ & & & & $0.754^{b}$ \\
\hline A & $18(56.3)$ & $8(50.0)$ & $10(62.5)$ & \\
\hline B & $9(28.1)$ & $5(31.2)$ & $4(25.0)$ & \\
\hline C & $5(15.6)$ & $3(18.8)$ & $2(12.5)$ & \\
\hline
\end{tabular}

aMann-Whitney $U$ test; ${ }^{\text {b}} \mathrm{Chi}$-squared test or Fischer exact test, when appropriate Table 1: Baseline demographics data and clinical characteristics of patient are separately for each treatment groups.
Blood samples were collected at the first clinic examination (T0), at 1 month (T1), at 3 months (T3), at six months (T6) and at twelve months (T12), to analyse plasma levels of glucose, triglycerides, total cholesterol, high-density lipoprotein (HDL)-cholesterol, low-density lipoprotein (LDL)-cholesterol, aspartate aminotransferases (AST), alanine aminotransferases (ALT) and creatinine. The study was conducted in agreement with the Helsinki declaration. At the enrolment, all subjects provided a written informed consent. Statistical analysis was performed with SPSS software 20.0 (SPSS Inc., Chicago, IL, USA). Linear mixedeffect models for repeated measurements were applied to analyze the effect of treatment on continuous outcome variables. Models were used to regress pre- and post-treatment measures with patients as a random effect assuming unstructured covariance matrix. A Contrast analysis, $a$ priori specified, was also used to evaluate the difference between groups at each time point.

\section{Results}

Patients were randomized to the therapeutic regimen with RAL (group 1) or without RAL (group 2), with a computerized random allocation sequence, after the selection of the triple therapy on the basis of resistance testing. Patients were assigned to one of the two groups with an equal probability for each group. The induction therapy with RAL was made for six months. The baseline demographic and clinical characteristics of patients are detailed in Table 1. At baseline, the group 1 showed a significantly higher viral load than group $2(476,728 \pm 279,284$ copies/ml vs. 146,141 $\pm 39,265$ copies/ml, respectively; $\mathrm{p}=0.048)$. After one month of therapy (T1), the group 1 showed a better reduction of plasma HIV-RNA than group $2(259 \pm 88$ copies $/ \mathrm{ml} v s .1007 \pm 187 \mathrm{cp} /$ $\mathrm{ml}$, respectively; $\mathrm{p}<0.05)$. At the remaining time points, the reduction was comparable in group 1 and 2 (at T12 $7 \pm 5 \mathrm{cp} / \mathrm{ml} v s .7 \pm 2 \mathrm{cp} / \mathrm{ml}$, respectively) (Figure 1A). Based on ART therapy, significant differences in the immunological recovery were observed. The group 1 showed a higher CD4+ recovery than group 2 at T3 (T0 $272 \pm 49 \rightarrow$ T3 $542 \pm 90$ vs. T0 $264 \pm 32 \rightarrow \mathrm{T} 3396 \pm 44$, respectively; $\mathrm{p}<0.05$ ) (Figure 1B). On the contrary, no significant changes of metabolic parameters and of indexes of hepatic and renal function in both groups were found, except for a significant increase in total cholesterol and HDL cholesterol levels in both groups (Table 2), although always within the normal range. The four-drug regimen was safe and well tolerated, in the absence of any virological failure.
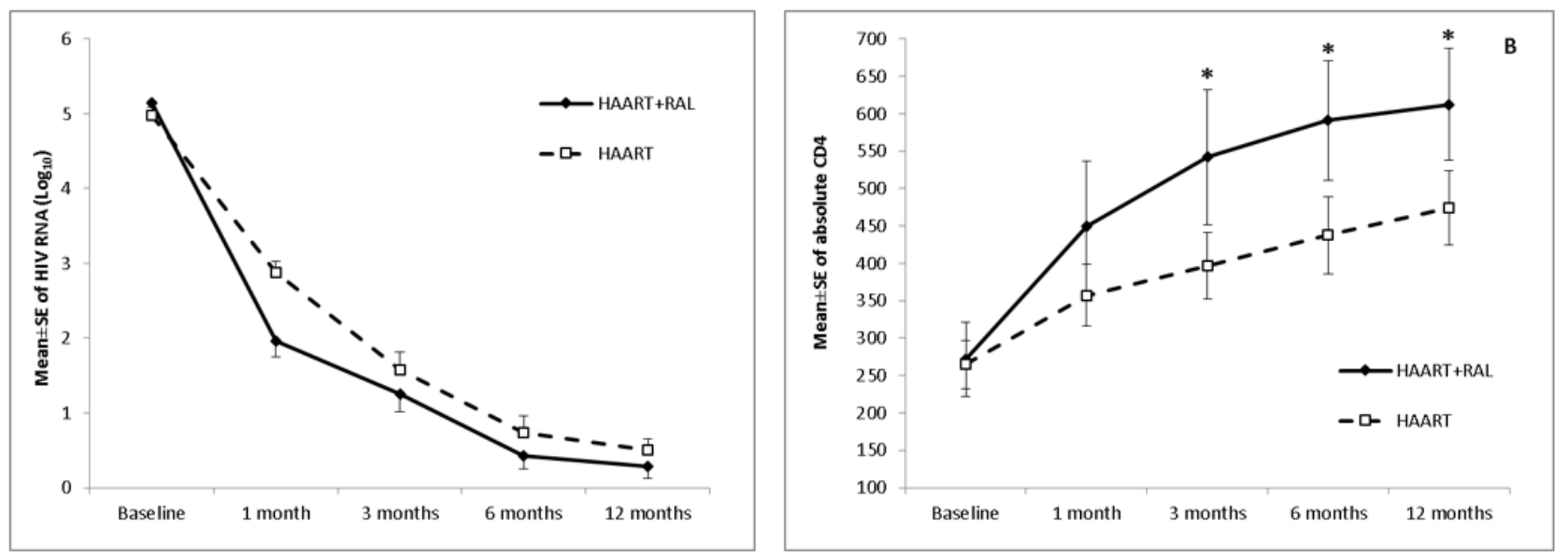

Figure 1: Mean and Standard error of HIVRNA (Panel A) and absolute CD4 (Panel B) seperately for two group of treatment at baseline and 1, 3, 6 and 12 months of follow-up. 
Citation: Falasca K, Ucciferri C, Nicola MD, Vignale F, Racciatti D, et al. (2017) A Four-Drug Induction Therapy Including Raltegravir for the Treatment of Naïve HIV-Infected Patients. J AIDS Clin Res 8: 705. doi: 10.4172/2155-6113.1000705

Page 3 of 4

\begin{tabular}{|c|c|c|c|c|c|c|c|c|c|c|c|}
\hline \multirow[t]{2}{*}{ Variable } & \multicolumn{4}{|c|}{ HAART+RAL group } & \multicolumn{4}{|c|}{ HAART group } & \multicolumn{3}{|c|}{$p$-value } \\
\hline & Baseline & 3 months & 6 months & 12 months & Baseline & 3 months & 6 months & 12 months & +time & *treatment & §interaction \\
\hline Triglycerides, mg/dL & $126.8 \pm 61.9$ & $136.1 \pm 69.3$ & $130.3 \pm 53.4$ & $133.2 \pm 41.7$ & $125.5 \pm 70.9$ & $143.9 \pm 61.9$ & $130.2 \pm 55.7$ & $137.4 \pm 64.3$ & 0.781 & 0.885 & 0.986 \\
\hline Total cholesterol, mg/dL & $164.4 \pm 32.0$ & $189.7 \pm 21.7$ & $188.5 \pm 28.1$ & $189.3 \pm 25.2$ & $160.1 \pm 23.6$ & $198.9 \pm 36.9$ & $187.7 \pm 37.7$ & $193.7 \pm 35.8$ & 0.001 & 0.261 & 0.332 \\
\hline HDL cholesterol, mg/dL & $40.2 \pm 11.8$ & $47.3 \pm 11.4$ & $50.0 \pm 12.0$ & $47.0 \pm 9.8$ & $44.7 \pm 13.8$ & $53.9 \pm 13.2$ & $51.1 \pm 13.4$ & $53.7 \pm 14.8$ & 0.001 & 0.722 & 0.392 \\
\hline LDL cholesterol, mg/dL & $98.2 \pm 28.6$ & $114.6 \pm 26.8$ & $112.1 \pm 23.9$ & $115.9 \pm 22.1$ & $89.1 \pm 22.2$ & $116.1 \pm 31.2$ & $112.7 \pm 31.9$ & $110.7 \pm 28.7$ & 0.095 & 0.805 & 0.434 \\
\hline Creatinine, $\mathrm{mg} / \mathrm{dL}$ & $0.81 \pm 0.13$ & $0.89 \pm 0.18$ & $0.89 \pm 0.17$ & $0.89 \pm 0.17$ & $0.87 \pm 0.19$ & $0.91 \pm 0.24$ & $0.88 \pm 0.22$ & $0.89 \pm 0.16$ & 0.224 & 0.193 & 0.622 \\
\hline GOT, U/L & $35.8 \pm 18.0$ & $29.4 \pm 10.6$ & $32.2 \pm 10.2$ & $30.9 \pm 8.1$ & $34.3 \pm 15.9$ & $27.5 \pm 6.8$ & $27.1 \pm 4.7$ & $27.7 \pm 6.2$ & 0.056 & 0.301 & 0.697 \\
\hline GPT, U/L & $49.9 \pm 61.2$ & $47.3 \pm 34.7$ & $42.5 \pm 18.5$ & $41.0 \pm 15.5$ & $36.9 \pm 20.3$ & $27.6 \pm 11.4$ & $25.1 \pm 11.9$ & $28.8 \pm 10.6$ & 0.673 & 0.486 & 0.626 \\
\hline Glicemia, mg/dL & $87.3 \pm 15.9$ & $84.6 \pm 16.5$ & $82.9 \pm 14.8$ & $84.3 \pm 12.9$ & $86.6 \pm 32.5$ & $80.2 \pm 8.5$ & $80.0 \pm 7.1$ & $79.0 \pm 7.6$ & 0.047 & 0.828 & 0.429 \\
\hline
\end{tabular}

time: the differences have been tested between the means of all times values of the two groups of treatment

*treatment: the differences have been tested between the means of each group of treatment at all times

interaction: probability that the effects of time is different in one distinct group of treatment (interaction time*treatment)

Table 2: Biochemical characteristics (mean \pm standard deviation) of HIV infected patients under HAART+RAL and HAART therapy at baseline and at follow-up.

\section{Discussion}

This study showed that a four-drug induction therapy with RAL is effective and safe in HIV infected patients, and ensures a better immune recovery likely correlated to a rapid decay of viremia. RAL is an HIV-1 integrase strand-transfer inhibitor approved for use in cART of HIV1 infection both in treatment-naïve and previously treated patients. Integrase inhibitors are unique among antiretroviral agents because of the specific interaction with two HIV critical elements the virally encoded integrase enzyme, and the HIV1 LTR [9]. This study allowed confirming that RAL is effective especially as first-choice drug in treatment-naïve patients due to a rapid drop of viral load, showing also a good safety profile because none of the patients had to discontinue the induction treatment due to adverse events or virological failure. With the advent of ART, plasma HIV-1 replication can become undetectable for years. However, HIV-1 can persist in lymphoid reservoirs where residual low levels of viral replication can be found [17]. Consequently, ART intensification may act on the residual HIV replication and on HIV reservoirs. Therapeutic regimens including maraviroc and raltegravir do not act on blood proviral HIV-DNA reservoirs, but can decrease the cell-associated HIV-RNA, the CD8+ T cells activation, and may have a possible impact on rectal proviral HIV DNA in some patients [18]. It is known that a rapid drop of the viral load correlates to a reduction of HIV concentrations, particularly in the reservoirs. Treatment intensification with RAL significantly decreases the latent cellular HIV reservoir, as well as the CD8+ T cell activation [19]. In this study, we found, for the first time, a rapid and significant improvement in CD4+ $\mathrm{T}$ cells count in patients with four drug induction therapies and a faster viral decay in this group. The rapid decay of viral load observed is very important; the significant reduction of viral load observed at $\mathrm{T} 1$ associated to RAL therapy could be correlated to a significant and persistent increase of $\mathrm{CD} 4+\mathrm{T}$ cells count, and this difference could persist over time, even after the end of RAL induction therapy. This increase might be due to the effect of intensification therapy, especially on the ongoing viral replication. Several clinical trials have argued on ART intensification, and the results were conflicting; no effects on the residual viremia measured by the single copy assay [20-22] or on immunological recovery [23] were reported. Some observational studies showed that at least $76 \%$ of HIV infected patients starting ART achieved an undetectable viral load within the first 6 months of therapy [24], while a percentage of $9 \%-45 \%$ did not obtain an appropriate recovery of CD4+ $\mathrm{T}$ cells count $[25,26]$. This condition, commonly known as immuno-virological discordance and associated with a lower CD4+ nadir, can lead to an increased risk of progression to AIDSdefining illness and death $[27,28]$. Furthermore, it has been argued that the lack of $\mathrm{CD} 4+\mathrm{T}$ cells recovery and the disease progression may be due to a persistent immune activation [29,30]. In this study, the fourdrug induction therapy with RAL resulted in a significant marked improvement in CD4+ T cells count. Unexpectedly, the patients group with RAL showed a lower impact on the increase in principal metabolic parameters compared to the group under standard cART.

Anyway, further extensive studies are needed to confirm our data, and to approve the use of a four-drug induction therapy with RAL in patients with high levels of viral load at baseline.

\section{Conclusion}

Intensification induction therapy with RAL for a period of six months in HIV infected treatment-naïve patients may have a significant advantage both in reconstituting CD4+ T cells and in decreasing the viral load in a faster way and this difference seems to be persistent over time even after RAL discontinuation.

\section{References}

1. Vecchiet J, lachininoto MG, Capodimonti S, Nuzzolo ER, Falasca K, et al. (2013) Effect of antiviral therapy on pro-angiogenic hematopoietic and endothelial progenitor cells in HIV-infected people. Thromb Res 131: 238-243.

2. Falasca K, Ucciferri C, Teofili L, lachininoto MG, Capodimonti S, et al. (2013) Short communication: Proangiogenic hematopoietic cells in acute HIV infection. AIDS Res Hum Retroviruses 29: 307-310.

3. Rodger AJ, Lodwick R, Schechter M, Deeks S, Amin J, et al. (2013) Mortality in well controlled HIV in the continuous antiretroviral therapy arms of the SMART and ESPRIT trials compared with the general population. AIDS 27: 973-979.

4. Fong R, Cheng AC, Vujovic O, Hoy JF (2013) Factors associated with virological failure in a cohort of combination antiretroviral therapy-treated patients managed at a tertiary referral centre. Sex Health 10: 442-447.

5. Hirsch MS, Gunthard HF, Schapiro JM, Brun-Vezinet F, Clotet B, et al (2008) Antiretroviral drug resistance testing in adult HIV-1 infection: 2008 recommendations of an International AIDS Society-USA panel. Clin Infect Dis 47: 266-285.

6. Kearney M, Spindler J, Shao W, Maldarelli F, Palmer S, et al. (2011) Genetic diversity of simian immunodeficiency virus encoding HIV-1 reverse transcriptase persists in macaques despite antiretroviral therapy. J Virol 85: 1067-1076.

7. Metzner KJ, Allers K, Rauch P, Harrer T (2007) Rapid selection of drug-resistant HIV-1 during the first months of suppressive ART in treatment-naive patients. AIDS 21: 703-711.

8. Metzner KJ, Giulieri SG, Knoepfel SA, Rauch P, Burgisser P, et al. (2009) Minority quasi species of drug-resistant HIV-1 that lead to early therapy failure in treatment-naive and -adherent patients. Clin Infect Dis 48: 239-247.

9. Hazuda DJ (2012) HIV integrase as a target for antiretroviral therapy. Curr Opin HIV AIDS 7: 383-389.

10. Sharma M, Walmsley SL (2014) Raltegravir as antiretroviral therapy in HIVI AIDS. Expert Opin Pharmacother 15: 395-405. 
Citation: Falasca K, Ucciferri C, Nicola MD, Vignale F, Racciatti D, et al. (2017) A Four-Drug Induction Therapy Including Raltegravir for the Treatment of Naïve HIV-Infected Patients. J AIDS Clin Res 8: 705. doi: 10.4172/2155-6113.1000705

11. Markowitz M, Nguyen BY, Gotuzzo E, Mendo F, Ratanasuwan W, et al. (2007) Rapid and durable antiretroviral effect of the HIV-1 Integrase inhibitor raltegravir as part of combination therapy in treatment-naive patients with HIV-1 infection: Results of a 48-week controlled study. J Acquir Immune Defic Syndr 46: 125133.

12. Noguera-Julian M, Casadella M, Pou C, Rodriguez C, Perez-Alvarez S, et al. (2013) Stable HIV-1 integrase diversity during initial HIV-1 RNA decay suggests complete blockade of plasma HIV-1 replication by effective raltegravircontaining salvage therapy. Virol J 10: 350

13. Sedaghat AR, Siliciano RF, Wilke CO (2008) Low-level HIV-1 replication and the dynamics of the resting CD4+ T cell reservoir for HIV-1 in the setting of HAART. BMC Infect Dis 8: 2 .

14. Lennox JL, DeJesus E, Lazzarin A, Pollard RB, Madruga JV, et al. (2009) Safety and efficacy of raltegravir-based versus efavirenz-based combination therapy in treatment-naive patients with HIV-1 infection: A multicentre, doubleblind randomised controlled trial. Lancet 374: 796-806.

15. Sedaghat AR, Dinoso JB, Shen L, Wilke CO, Siliciano RF (2008) Decay dynamics of HIV-1 depend on the inhibited stages of the viral life cycle. Proc Natl Acad Sci U S A 105: 4832-4837.

16. Lichtenstein KA, Armon C, Nagabhushanam V, Efaw BJ, Frazer-Abel A, et al (2012) A pilot study to assess inflammatory biomarker changes when raltegravir is added to a virologically suppressive HAART regimen in HIV-1-infected patients with limited immunological responses. Antivir Ther 17: 1301-1309.

17. Svicher V, Ceccherini-Silberstein F, Antinori A, Aquaro S, Perno CF (2014) Understanding HIV compartments and reservoirs. Curr HIVIAIDS Rep 11: 186-194.

18. Lafeuillade A, Assi A, Poggi C, Bresson-Cuquemelle C, Jullian E, et al. (2014) Failure of combined antiretroviral therapy intensification with maraviroc and raltegravir in chronically HIV-1 infected patients to reduce the viral reservoir: The IntensHIV randomized trial. AIDS Res Ther 11: 33.

19. Vallejo A, Gutierrez C, Hernandez-Novoa B, Diaz L, Madrid N, et al. (2012) The effect of intensification with raltegravir on the HIV-1 reservoir of latently infected memory CD4 T cells in suppressed patients. AIDS 26: 1885-1894.

20. Gandhi RT, Zheng L, Bosch RJ, Chan ES, Margolis DM, et al. (2010) The effect of raltegravir intensification on low-level residual viremia in HIV-infected patients on antiretroviral therapy: A randomized controlled trial. PLoS Med 7.
21. Buzon MJ, Massanella M, Llibre JM, Esteve A, Dahl V, et al. (2010) HIV-1 replication and immune dynamics are affected by raltegravir intensification of HAART-suppressed subjects. Nat Med 16: 460-465.

22. Dinoso JB, Kim SY, Wiegand AM, Palmer SE, Gange SJ, et al. (2009) Treatment intensification does not reduce residual HIV-1 viremia in patients on highly active antiretroviral therapy. Proc Natl Acad Sci U S A 106: 9403-9408.

23. Rusconi S, Vitiello P, Adorni F, Colella E, Foca E, et al. (2013) Maraviroc as intensification strategy in HIV-1 positive patients with deficient immunological response: An Italian randomized clinical trial. PLoS ONE 8: e80157.

24. Street E, Curtis H, Sabin CA, Monteiro EF, Johnson MA (2009) British HIV Association (BHIVA) national cohort outcomes audit of patients commencing antiretrovirals from naive. HIV Med 10: 337-342.

25. Tan $\mathrm{R}$, Westfall AO, Willig JH, Mugavero MJ, Saag MS, et al. (2008) Clinical outcome of HIV-infected antiretroviral-naive patients with discordant immunologic and virologic responses to highly active antiretroviral therapy. $J$ Acquir Immune Defic Syndr 47: 553-558.

26. Robbins GK, Spritzler JG, Chan ES, Asmuth DM, Gandhi RT, et al. (2009) Incomplete reconstitution of $\mathrm{T}$ cell subsets on combination antiretroviral therapy in the AIDS Clinical Trials Group protocol 384. Clin Infect Dis 48: 350-361.

27. HIV Surrogate Marker Collaborative Group (2000) Human immunodeficiency virus type 1 RNA level and CD4 count as prognostic markers and surrogate end points: A meta-analysis. AIDS Res Hum Retroviruses 16: 1123-1133.

28. d'Arminio Monforte A, Testori V, Adorni F, Castelnuovo B, Bini T, et al. (1999) CD4 cell counts at the third month of HAART may predict clinical failure. AIDS 13: $1669-1676$.

29. Giorgi JV, Hultin LE, McKeating JA, Johnson TD, Owens B, et al. (1999) Shorter survival in advanced human immunodeficiency virus type 1 infection is more closely associated with $\mathrm{T}$ lymphocyte activation than with plasma virus burden or virus chemokine coreceptor usage. J Infect Dis 179: 859-870.

30. Marchetti G, Gori A, Casabianca A, Magnani M, Franzetti F, et al. (2006) Comparative analysis of T-cell turnover and homeostatic parameters in HIVinfected patients with discordant immune-virological responses to HAART. AIDS 20: 1727-1736. 